The duodenal ampulla was reliably identified in 3/47 (6.4\%) examinations. Pathology was identified in $11 / 47$ (23.4\%) examinations.

Conclusion Side-viewing VCE was well tolerated and completed examination results were available for $76.4 \%$ of patients examined. Our findings did not correlate with previous reported results $(71 \%)$ regarding the identification rate of the duodenal papilla as a small bowel landmark with side-viewing VCE.

Advantages of the side-viewing VCE are not needing the patient to wear a recorder, with the data being stored in the capsule itself. This enables multiple patients to be examined on the same day and the number of examinations is not limited by available data recorders. Patients can also take the capsule home and take the capsule at any time which can be useful in the investigation of obscure GI bleeding. Side-viewing VCE is comparable to forward viewing VCE with respect to cost and accuracy.

Disclosure of Interest None Declared.

\section{PTU-045 FLEXIBLE ENDOSCOPIC TREATMENT OF ZENKER'S DIVERTICULUM USING A SOFT DIVERTICULOSCOPE AND AN ENDOSCOPIC KNIFE - VIDEO ABSTRACT}

R Bhattacharyya*, P Bhandari on behalf of Portsmouth research group. Gastroenterology, Portsmouth Hospitals NHS Trust, Cosham, Portsmouth, UK

\subsection{6/gutjnl-2014-307263.119}

Introduction Zenker's diverticulum is a mucosal outpouching through the posterior pharyngeal wall resulting from increased hypopharyngeal pressure. Symptoms may include dysphagia and regurgitation of food. Treatment consists of myotomy of the cricopharyngeus muscle, which is commonly done through an endosurgical approach using a rigid endoscope. We describe a novel method of endoscopic diverticulotomy using a flexible diverticuloscope and an endoscopic knife.

Methods With the patient under general anaesthesia, a guidewire is inserted under direct vision into the oesophageal lumen through a gastroscope. The gastroscope is removed and the guidewire is threaded through a hole on the long flap of the flexible diverticuloscope (ZD overtube, ZDO-22 _ 30; Cook Endoscopy, Winston-Salem, North Carolina). The overtube is gently advanced to $20 \mathrm{~cm}$ until resistance is felt. The gastroscope is then inserted through the overtube and its position adjusted so that the longer flap is in the true oesophageal lumen and the shorter flap is in the diverticulum. The septum is now clearly visualised and stable between the two flaps.

A $3 \mathrm{~mm}$ needle-knife is used to incise the septum. The cut is performed until the muscle fibres are completely cut, and then the cut is extended to a section of the anterior diverticulum and posterior oesophageal wall up to approximately $1 \mathrm{~cm}$ from the bottom. At the end of the procedure, one to three endoclips are placed to prevent perforation or bleeding.

Results We have performed this procedure in 3 patients. 1 patient had no previous surgical intervention, 1 had recurrence following previous surgical diverticulotomy, and 1 patient had severe cervical spondylosis due to which surgery could not be performed as adequate extension of the neck for the surgical procedure was not possible. There have been no complications. Conclusion Endoscopic Zenker's diverticulotomy can be safely performed using a flexible diverticuloscope. We demonstrate the feasibility of performing endoscopic diverticulotomy in patients with recurrence after surgery, or in those who are unsuitable for surgery. We aim to illustrate the principles and technique of this procedure by video demonstration.

Disclosure of Interest None Declared.

\section{PTU-046 MIND OVER MATTER? GASTROINTESTINAL BLEEDING DUE TO THE USE OF NOVEL ORAL ANTICOAGULANTS IN STROKE PREVENTION}

${ }^{1} \mathrm{~S}$ Beg*, '1 Patel, 'M Bhuva, ${ }^{2} \mathrm{D}$ Collas, ${ }^{1} \mathrm{~B}$ Macfarlane. 'Gastroenetrology, Watford General Hospital, Hertfordshire, UK; ${ }^{2}$ Stroke Medicine, Watford General Hospital, Hertfordshire, UK

\subsection{6/gutjnl-2014-307263.120}

Introduction The occurrence of stroke secondary to the prothrombotic effects of AF is well recognised, with warfarinisation the established therapy for prevention. This is not without complications and so the introduction of the novel oral anticoagulants (NOAC), Dabigatran a factor IIa inhibitor and Rivaroxaban a Xa inhibitor have been received with enthusiasm. Advantages include single dosing, fewer drug interactions and freedom from monitoring, whilst data has demonstrated non-inferiority with respects to stroke prevention. A reported greater propensity to gastrointestinal (GI) bleeding is concerning given the lack of accepted reversal agents, in a cohort of patients who tend to be elderly with comorbidities. We aim to establish the GI complications associated with NOACs in stroke prevention.

Methods We retrospectively analysed 170 consecutive patients from our TIA clinic, who were found to have AF. They were commenced on either Dabigatran or Rivaroxaban, with a total of 86 patient-years of exposure at the time of analysis. We interrogated our computer-based database to ascertain the incidence of anticoagulation related admissions and subsequent management

Results Our cohort of 170 patients had a mean age of 76 years, with a male to female ratio of 8:9. Dabigatran at $220-300 \mathrm{mg} /$ day was prescribed to $45 \%(\mathrm{n}=77)$, whilst $55 \%(\mathrm{n}=93)$ received Rivaroxaban 20-15 mg/day. Rivaroxaban was stopped in one patient due to drug induced hepatitis. One patient was switched from Dabigatran to Rivaroxaban due to dyspepsia.

GI bleeds occurred in $5 \%(\mathrm{n}=4)$ of the Dabigatran group, with 2 upper and 2 lower GI bleeds, occurring on average after 25 days. These patients had a mean age of 84 years, with at least 2 comorbidities. Endoscopy revealed an oesophageal ulcer requiring clipping, new diagnosis of colorectal cancer, haemorrhoids and in one patient no cause was found. GI bleeding occurred in $1 \%(\mathrm{n}=1)$ of the Rivaroxaban group. The patient had a rectal bleed, was 96 years and too frail for investigation, therapy was stopped. The average blood transfusion requirement was 4 units. One individual required resuscitation with 16 units of blood, 12 of fresh frozen plasma, 4 of cryoprecipitate and 1 pool of platelets. Average length of admission was 8.4 days.

Conclusion Existing data suggests that the GI bleed rate whilst on warfarin ranges from $1.3-2.6 \%$, compared to a rate of $2.3-$ $3.6 \%$ in association with NOACs. In our series there was an overall GI bleed rate of $3 \%$, with $1.8 \%$ that could be considered to be significant, requiring blood products and cessation of anticoagulation. Should NOACs become more widely used the lack of established reversal techniques will require local GI units to provide a prompt and skilled bleed service.

Disclosure of Interest None Declared. 


\section{PTU-047 BIODEGRADABLE OESOPHAGEAL STENTS IN BENIGN AND MALIGNANT DISEASE - A SINGLE CENTRE EXPERIENCE}

${ }^{1} S S$ Siddhi*, ${ }^{2}$ J Plevris, 'S Bow. 'Gastroenterology, Royal Infirmary Edinburgh, UK; ${ }^{2}$ Gastroenterology, Royal Infirmary Edinburgh, University of Edinburgh, Edinburgh, UK

10.1136/gutjnl-2014-307263.121

Introduction Biodegradable oesophageal stents have been developed recently and the experience in their use and available literature in limited. Their usefulness has been demonstrated in refractory benign strictures in a handful of studies and their role in malignant strictures is relatively untested. We looked at our practice and the clinical outcomes in the use of these stents.

Methods This is a retrospective observational study looking at electronic case record and endoscopy reports. All patients who had biodegradable stents inserted between March 2011 and September 2013 were included for analysis.

Twenty-three stents were inserted in 16 patients. 5 of these patients had 2 stents and one patient had 3 stents over the period of time. Thirteen stents were inserted for benign disease and eight for malignant strictures. There were 9 males and 7 females with age range being between $47 \mathrm{yrs}$ and $101 \mathrm{yrs} 2$ stents were inserted in 1 patient with postoperative tracheooesophageal fistula.

Results In the benign group, an average of 10.5 endoscopies $(0.95 / \mathrm{month} /$ patient $)$ and 7.2 dilatations $(0.65 / \mathrm{month} /$ patient $)$ were necessary prior to stent insertion per patient. Post insertion there was a reduction to 3.5 endoscopies $(0.03 /$ month/patient) and 2 dilatations $(0.016 / \mathrm{month} /$ patient). When the average number of dilatations was analysed pre and post stent insertions per patient per month, there was a significant reduction with a $\mathrm{P}$ value of 0.009 on the paired $\mathrm{T}$ test. In the benign group, on an average 7 endoscopies and 5 dilatations were avoided/patient. Interval between dilatations increased from 5.2 to 25 weeks.

In the malignant group, all patients were successfully bridged to chemo/radiotherapy. 2 out 6 patients went on to metal stents after 3 and 5 months due to disease progression.

The stent insertion was technically successful in all cases following a dilatation of the stricture to $12 \mathrm{~mm}$ at the time of insertion. Four patients complained of some pain post stent insertion ( 3 in the malignant group and 1 in the benign group). One patient developed sepsis post procedure but recovered well with a course of antibiotics. No other complications were noted. In all cases where a check up endoscopy was done, the stent had disintegrated within 8-12 weeks.

Conclusion BD stents are of value in recurrent benign oesophageal strictures as they reduced the number and frequency of dilatations. The BD stents successfully bridge patients with malignant strictures to oncological therapies.

Insertion of a BD stent does not preclude a later insertion of a permanent metal stent. Stent insertion was technically successful and safe.

Disclosure of Interest None Declared.

\section{PTU-048 INITIAL EXPERIENCE WITH RADIOFREQUENCY ABLATION IN GASTRIC ANTRAL VASCULAR ECTASIA AND RADIATION PROCTITIS}

SS Siddhi* ${ }^{*}$ H Dalziel, EB Henry. Gastroenterology, Ninewells Hospital and Medical School, NHS Tayside, Dundee, UK

10.1136/gutjnl-2014-307263.122
Introduction Gastric Antral Vascular Ectasia (GAVE) and radiation proctitis can cause chronic GI bleeding and may be severe enough to cause transfusion dependent anaemia.

Current standard therapy for both these conditions is endoscopy with argon plasma coagulation. This usually requires multiple endoscopies over time and in many patients may be ineffective.

With the development of Radiofrequency Ablation (RFA) in Barrett's Oesophagus, we extended its use in these two settings. The available literature is small and our study adds to this body of evidence.

Methods Retrospective case record study. We collected data from all patients who received RFA between December 2012 and November 2013. Data was collected from endoscopy reports and electronic case records.

RFA was performed using the Halo 60,90 or through the scope probes at $12 \mathrm{j}$ energy (Barxx/Covidien).

Results Three patients received RFA for GAVE and six patients for Radiation proctitis.

GAVE: All patients presented with transfusion dependent anaemia. They needed $0.85 \mathrm{OGD} / \mathrm{pt} / \mathrm{month}, 0.41 \mathrm{APC} / \mathrm{pt} / \mathrm{month}$ and 6 units of packed cells /pt/month prior to RFA. Between 24 sessions of RFA was required. Post RFA, there was a reduction in endoscopies to $0.34 / \mathrm{pt} /$ month (P 0.239) In two out of three patients no further transfusions were required. Hence there was a significant reduction in transfusion requirement. (P 0.033).

Radiation Proctitis: All patients presented with PR bleeding. One was transfusion dependent. A mean of 0.58 APC sessions were done per patient/month. There was a significant reduction in the number of sigmoidoscopies from $0.87 / \mathrm{pt} / \mathrm{month}$ to $0.2 / \mathrm{pt} /$ month (P 0.007). One patient who was requiring 9 units of packed cells per month stopped transfusions after RFA. Number of RFA sessions was between 1-3 (mean: 1.66).

In the NHS, therapeutic sigmoidoscopy with APC costs $£ 704$ and therapeutic OGD is $£ 667$. A single unit of blood transfusion costs $£ 635$. An RFA probe costs $£ 920$ and hence an RFA procedure proves cost effective above $2 \mathrm{x}$ APC or $2 \mathrm{x}$ blood transfusions.

Our only complication was one clinically insignificant stricturing but this was after $8 \mathrm{xAPC}$ and $3 \mathrm{X}$ RFA. Healing is optimum after 3 months. It is better tolerated than APC in terms of comfort.

Conclusion RFA for GAVE and RP is technically feasible, well tolerated and cost effective. We have demonstrated that there was a significant reduction in blood transfusions in GAVE and requirement for Sigmoidoscopy in RP.

We have shown no significant complications and RFA should be considered as a first line treatment in refractory GAVE and in $\mathrm{RP}$ where bleeding is significant.

We recognise this is a small, retrospective study but future work will include larger numbers, QoL data and establish if this should be considered the first line therapy.

Disclosure of Interest None Declared.

\section{PTU-049 HOW REWARDING IS GASTROSCOPY IN DIAGNOSIS OF CANCER IN ISOLATED IRON DEFICIENCY ANAEMIA?}

SK Butt* ${ }^{*}$ K Besherdas. Gastroenterology, Chase Farm Hospital, London, UK

\subsection{6/gutjnl-2014-307263.123}

Introduction The ultimate goal of the UK Cancer plan is to 'offer patients a maximum one month wait from an urgent referral for suspected cancer to the beginning of treatment'. The North 